\title{
Expanding Our Scope to Include Biomechanics Education and Outreach
}

There has been growing interest in establishing and sharing best practices to improve the education and outreach of biomechanics. Many of the researchers in the field of biomechanics are educators themselves, whether formally serving as course instructors or being active in biomechanics outreach. An increasing number of these educators have begun to apply their research skills to rigorously evaluate the effectiveness of their teaching pedagogies, as well as the specific approaches, activities, and learning innovations they implement. While there is currently no central hub for disseminating these findings, an increase in education-related conference presentations and publications in various journals has been observed. Such work is important because it informs the field and helps educators teach more effectively so that student learning and outcomes can be maximized.

In this inaugural section, Journal of Applied Biomechanics is expanding its scope to begin publishing papers focused on the education and outreach of biomechanics. Such papers will be considered across all scales including: global or national initiatives; community partnerships and programs; interdisciplinary university programs; biomechanics curriculum; specific course structure or approach; teaching pedagogies; and specific approaches, activities, and learning innovations. In addition to more traditional educational experiences, papers focused on topics such as graduate student mentorship, undergraduate research experiences, scientific literacy, responsible conduct of research, and scientific advocacy will also be considered. More details can be found in the Author Guidelines on the journal website.

After this issue, we anticipate publishing such papers regularly. While the vast majority of papers published in Journal of Applied Biomechanics will continue to be studies of human movement, we hope that adding education-related papers will continue to advance the impact of the journal on the field.

Kimberly Bigelow, Associate Editor Michael L. Madigan, Editor-in-Chief 